\title{
Evaluation of the diagnostic performance of infrared imaging of the breast: a preliminary study
}

\author{
Jane Wang ${ }^{1,2}$, King-Jen Chang ${ }^{3}$, Chin-Yu Chen ${ }^{4}$, Kuo-Liong Chien ${ }^{5}$, Yuh-Show Tsai ${ }^{6}$, Yuh-Ming Wu ${ }^{7}$, \\ Yu-Chuan Teng ${ }^{1}$, Tiffany Ting-Fang Shih ${ }^{1,2^{*}}$
}

\author{
* Correspondence: \\ ttfshih@ntu.edu.tw \\ 'Department of Medical Imaging, \\ National Taiwan University Hospital, \\ 7 Chung-Shan South Road, Taipei \\ 100, Taiwan
}

\begin{abstract}
Background: The study was conducted to investigate the diagnostic performance of infrared (IR) imaging of the breast using an interpretive model derived from a scoring system.

Methods: The study was approved by the Institutional Review Board of our hospital. A total of 276 women (mean age $=50.8$ years, SD 11.8) with suspicious findings on mammograms or ultrasound received IR imaging of the breast before excisional biopsy. The interpreting radiologists scored the lesions using a scoring system that combines five IR signs. The ROC (receiver operating characteristic) curve and AUC (area under the ROC curve) were analyzed by the univariate logistic regression model for each IR sign and an age-adjusted multivariate logistic regression model including 5 IR signs. The cut-off values and corresponding sensitivity, specificity, Youden's Index (Index = sensitivity+specificity-1), positive predictive value (PPV), negative predictive value (NPV) were estimated from the age-adjusted multivariate model. The most optimal cut-off value was determined by the one with highest Youden's Index.

Results: For the univariate model, the AUC of the ROC curve from five IR signs ranged from 0.557 to 0.701 , and the AUC of the ROC from the age-adjusted multivariate model was 0.828 . From the ROC derived from the multivariate model, the sensitivity of the most optimal cut-off value would be $72.4 \%$ with the corresponding specificity 76.6\% (Youden's Index = 0.49), PPV 81.3\% and NPV 66.4\%.
\end{abstract}

Conclusions: We established an interpretive age-adjusted multivariate model for IR imaging of the breast. The cut-off values and the corresponding sensitivity and specificity can be inferred from the model in a subpopulation for diagnostic purpose.

Trial Registration: NCT00166998.

\section{Background}

Infrared (IR) imaging of the breast, also known as breast thermography, is a non-invasive, painless examination which does not expose the subject to ionizing radiation, and is mainly a test of physiologic response of the breast findings [1-6]. It is based on the mechanism that the skin temperature overlying a malignancy is higher than skin overlying normal breast tissue. This is due to increasing infrared radiation and is most likely caused by elevated blood flow, metabolic activity, and angiogenesis at and around the lesion site $[2,5]$. IR imaging has been used for breast cancer detection since the

(c) 2010 Wang et al; licensee BioMed Central Ltd. This is an Open Access article distributed under the terms of the Creative Commons Attribution License (http://creativecommons.org/licenses/by/2.0), which permits unrestricted use, distribution, and reproduction in any medium, provided the original work is properly cited. 
1970s [1,2]. A nationwide study, Breast Cancer Detection Demonstration Projects (BCDDP) launched in 1973, investigated breast cancer screening by clinical breast examination, mammography and IR imaging. However, IR imaging was dropped at an early stage of the project due to unsatisfactory results [1-4]. This may have been due to technical difficulties, widely variable and subjective interpretation among image readers, unacceptably high false-positive and false-negative rates, and no direct aid for spatial localization of surgery [1-4]. However, abnormal findings on IR imaging of the breast were reported to be a risk factor and useful prognostic predictor for breast cancer, and IR imaging can also be an aid in the differential diagnosis of benign from malignant tumors $[1,3,6-10]$. The aforementioned values can be facilitated by modern computerized IR technology [1,3,6,7,10-12]. On the other hand, the diagnostic criteria varied among studies and the diagnostic performance, including sensitivity and specificity, also varied. Herein, we investigated the diagnostic performance of computerized breast IR imaging using an integrated interpretative model for breast IR imaging.

\section{Methods}

\section{Patients}

The study was approved by the Institutional Review Board of our hospital, and all study participants signed informed consents before the study. We enrolled 276 women (ages 17-81 years, mean $=50.8$ years, SD $=11.8$ years, median 50 years) who were scheduled to undergo excisional biopsy for suspicious findings on mammograms or ultrasound, or both. Patients with probably benign findings on mammography or ultrasound but received excisional biopsy due to surgeons' concerns were also included. IR imaging of the breast was done 1 day before surgery. Before enrolling subjects into this study, we excluded women who had a past history of breast surgery or chest irradiation, or systemic chemotherapy. The participants had to refrain from smoking, alcohol drinking, vigorous exercise, and application of lotion to the breasts within 4 hours before the procedure. Patients who received fine needle aspiration within 2 days or core needle breast biopsy within 2 weeks or any of who received vacuum-assisted breast biopsy before the study were also excluded.

\section{Procedures}

Computerized IR examination was done by two trained female radiological technicians using a medical thermographic system (ATIR-M301 Thermal Imaging System, Associated Technology Corporation, Chongqing, Sichuan, PROC), which was an uncooled micro-bolometer with focal plane array detector, and the image matrix size was $320 \times$ 240 with 14-bit depth, the pixel size was $45 \times 45 \mu \mathrm{m}$ with the response wave length $8-12 \mu \mathrm{m}$, and the temperature resolution was less than $0.1^{\circ} \mathrm{C}$. The procedure was done in a temperature controlled room maintained between 23 and $25^{\circ} \mathrm{C}$. Each participant was asked to disrobe and sit on a chair in an erect position, with hands over the head, sitting a distance about 2.5 meters away from the IR camera. After a total of $15 \mathrm{~min}$ utes rest, frontal, two true lateral (left and right lateral) and two oblique (left and right oblique) views of IR images were taken.

\section{Imaging processing and interpretation}

The images were viewed with a dedicated software program (M301-APP-V2.0, Associated Technology Corp., Chongqing, Sichuan, PROC) with manual brightness and 
contrast adjustment, and were displayed with either a gray-scale or a preset coloredscale.

A radiological technician and a radiologist (first radiologist) marked the lesion location and size of the lesions of concern, based on conventional imaging modalities including mammography, ultrasound, or both. They recorded the above information for each lesion on a sheet for the reference of the interpreting radiologists. Other two radiologists (second and third radiologists) were assigned to interpret the IR images; each read half of the cases. They interpreted the assigned IR images based only on the information of the above-mentioned sheet. The detailed mammographic and ultrasonographic, final pathologic findings of the study cases were only known to the first radiologist and were not available to the two IR imaging readers. The two radiologists who interpreted the IR images were both specialized in the field of breast imaging for more than 10 years.

The interpreting radiologists read the IR images based only on the findings at the lesion sites of concern and scored the findings according to the five independently diagnostic IR signs modified from the Ville Marie Infrared (IR) grading scale [7] and other reported literature [13-15]. The readers then recorded individual scores for each diagnostic IR sign for each lesion. We defined the IR signs as follows (Figures 1, 2, 3, $\& 4)$ :

IR1: a difference in surface temperature $(\mathrm{dT})$ at the lesion site from that at the mirror image site on the contralateral breast; the IR1 scale was scored as 0 for $\mathrm{dT}<=1^{\circ} \mathrm{C}$, as 1 for $1^{\circ} \mathrm{C}<\mathrm{dT}<=2^{\circ} \mathrm{C}$, and as 2 for $\mathrm{dT}>2^{\circ} \mathrm{C}$.

IR2: the $\mathrm{dT}$ between the lesion site and the rest of the normal breast tissue of the ipsilateral breast; the IR2 scale was scored as 0 for $\mathrm{dT}<=1^{\circ} \mathrm{C}$, and as 1 for $\mathrm{dT}>1^{\circ} \mathrm{C}$.

IR3: a combination of 8 various abnormal vascular patterns, including star vessel, inverted V vessel, fragmented vessel, closed vessel, vascular completeness, pointed or bifurcated vessels, moa-moa vascular pattern, or transverse vascular pattern [14]. Of them, the star vessels indicated vessels with radiating pattern and star shape; the fragmented vessels indicated that fragmented vascular anarchy in a localized area of the breast; closed vessel indicated vascular anarchy arranged in a closed pattern without evident branching; pointed or bifurcated vessels indicated vessels with pointed or

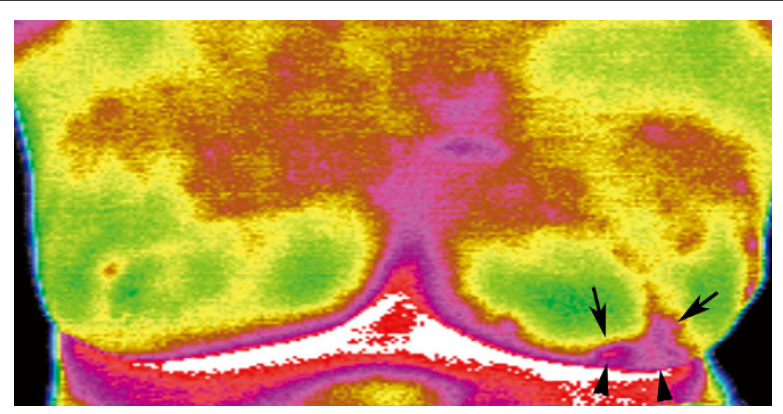

Figure 1 A 76-year-old woman with left breast cancer. IR imaging reveals focal increased surface temperature (positive IR1 sign with $\mathrm{dT}=1.5^{\circ} \mathrm{C}$ compared with the contralateral mirror image site; positive IR2 sign with $\mathrm{dT}=2^{\circ} \mathrm{C}$ compared with the remaining breast tissue at the ipsilateral side), abnormal vascular pattern (IR3 signs including closed vascular pattern, and vascular completeness) (arrows) and asymmetric vascular pattern (IR5 sign), and subtle focal bulging with back heat (IR4 sign) in left lower breast (arrowheads). Surgical pathological finding revealed a $4 \mathrm{~cm}$ infiltrating ductal carcinoma. 


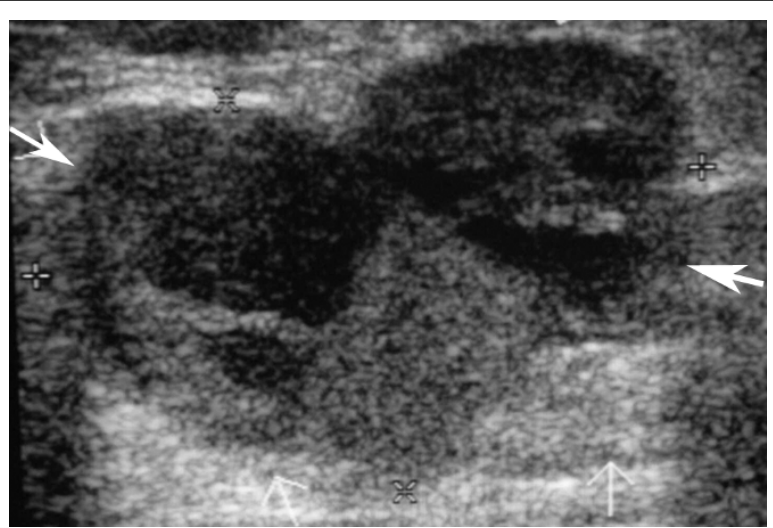

Figure 2 The corresponding breast ultrasound of the patient in Figure 1 shows a lobular mass at left lower breast, measuring about $3.1 \times 2.1 \mathrm{~cm}$ in diameter with heterogeneous echogenicity (arrows).

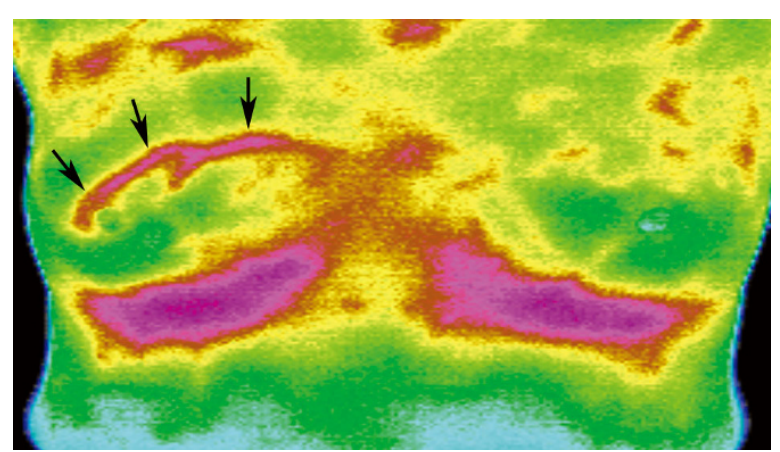

Figure 3 A 48-year-old woman with right breast cancer. IR imaging shows abnormally increased focal surface temperature (positive IR 1 sign with $\mathrm{dT}=1.2^{\circ} \mathrm{C}$ compared with the contralateral mirror image site; positive IR2 sign with $\mathrm{dT}=2^{\circ} \mathrm{C}$ compared with the remaining breast tissue in the ipsilateral breast), abnormal vascular pattern (IR3 signs including bifurcated vascular pattern, transverse vascular pattern, vascular completeness) and an asymmetric vascular pattern (IR5) in the right upper breast (arrows). The In $(\mathrm{OD})$ value for this finding scored by the interpreting radiologist was: $-5.463+0.0872(48)+0.3783(1)+1.9157(0)$ $+0.1728(1)+0.1578(3)+1.0278(0)+1.0363(1)=0.7834$, which is higher than the most optimal cut-off point (0.30) we selected in Table 4, and this is test-positive based on this cut-off point. (The radiologist scored the IR1 scale for this lesion as 1 , therefore, the $\mathrm{IR} 1_{\mathrm{A}}=1$ and $\mathrm{IR} 1_{\mathrm{B}}=0$ ).

bifurcated ends; moa-moa sign indicated a focal area of abnormal vascular pattern with irregular and engorged vascular branching with its shape mimicking a moa; transverse vascular pattern indicated a vessel which traverses part of the breast with a relatively somewhat horizontal or transverse orientation [14]. The presence of any of the above signs was scored as 1 and the absence of such signs as 0 . The sum of the eight signs was the score for the IR3 sign, which ranged from 0 to 8 and was treated as a continuous variable.

IR4: an edge sign or bulge sign backed by heat, indicating loss of smooth contour of part of the breast due to skin retraction or bulging caused by a breast tumor [16], the IR4 was scored as 0 when the sign was absent and as 1 when the sign was present.

IR5: the presence of an asymmetric or heterogeneous vascular pattern at and around the lesion site, when the contralateral breast did not reveal such a pattern. The IR5 scale was scored as 0 if the sign was absent and 1 if the sign was present. 


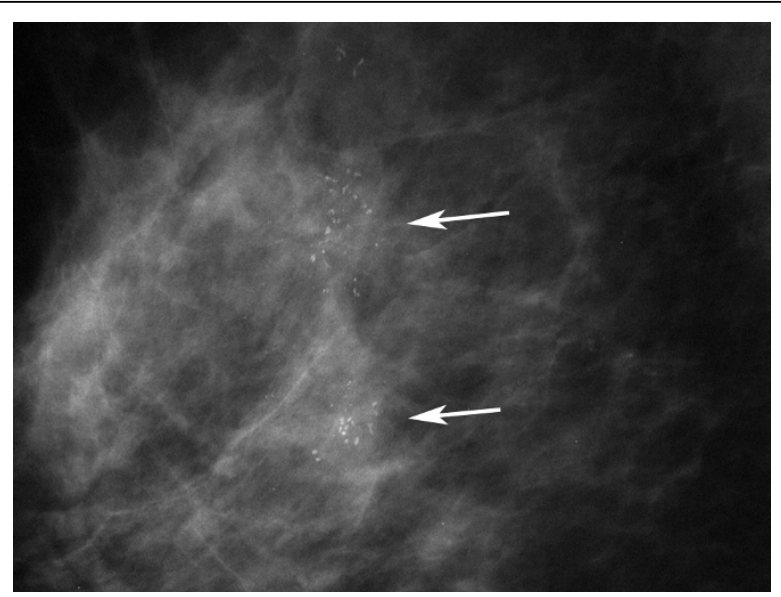

Figure 4 The right magnified mammogram of the patient in Figure 3 shows segmental pleomorphic microcalcifications in right upper breast (arrows).

\section{Statistical Analysis}

We analyzed the correlation of the diagnostic IR signs with the final disease status of the lesions at surgery using univariate and multivariate logistic regression analyses by SAS software (SAS version 9.00, SAS Institute, Cary, NC, USA) and SPSS software (SPSS version 16.0, SPSS Inc., Chicago, IL, USA). We categorized the final disease status as a dichotomous variable, that is, benign (including high risk lesions) or malignant. The high-risk lesions in our study included ADH (atypical ductal hyperplasia), ALH (atypical lobular hyperplasia), and papillary lesions.

For the univariate logistic regression analysis, we investigated the association of each individual IR sign with the final disease status. For the multivariate regression model, we put the five IR signs of each lesion into the model with age adjustment. The AUC (area under the ROC curve) values of the ROC (receiver operating characteristic) curves for each IR sign from the univariate model and for the age-adjusted multivariate model were estimated in order to investigate the diagnostic performance of the IR imaging. The Hosmer and Lemeshow test was applied to inspect the goodness-of-fit of the age-adjusted multivariate model, and a non-significant $p$ value $(>0.05)$ implies that the model is a fitted model. The age-adjusted multivariate model was presented as shown below:

$$
\ln [\mathrm{P} /(1-\mathrm{P})]=\ln (\text { odds })=\alpha+\beta_{0}(\text { Age })+\beta_{1 \mathrm{~A}}\left(\mathrm{IR}_{\mathrm{A}}\right)+\beta_{1 \mathrm{~B}}\left(\mathrm{IR}_{\mathrm{B}}\right)+\beta_{2}(\mathrm{IR} 2)+\beta_{3}(\text { IR3 })+\beta_{4}(\text { IR4 })+\beta_{5}(\text { IR5 })
$$

Where $\ln$ indicated the natural logarithm. The $\mathrm{P}$ value indicated the predicted probability of a lesion being malignant estimated from the regression model. Therefore, the $\mathrm{P} /(1-\mathrm{P})$ ratio indicated the odds (OD), that is, the ratio of the probability of being malignant to being benign for a given lesion. $\alpha$ is the intercept of the model. However, since IR1 is a trichotomous categorical variable, we re-coded the IR1 sign as a dummy variable for computations in logistic regression. That is:

if the IR1 scale of a given lesion was $0\left(\mathrm{dT}<=1^{\circ} \mathrm{C}\right)$, then the $\left(\operatorname{IR} 1_{\mathrm{A}}, \operatorname{IR} 1_{\mathrm{B}}\right)=(0,0)$;

if the IR1 scale was $1\left(1^{\circ} \mathrm{C}<\mathrm{dT}<=2^{\circ} \mathrm{C}\right)$, then the $\left(\operatorname{IR} 1_{\mathrm{A}}, \operatorname{IR} 1_{\mathrm{B}}\right)=(1,0)$;

if the IR1 scale was $2\left(\mathrm{dT}>2^{\circ} \mathrm{C}\right)$, then the $\left(\operatorname{IR} 1_{\mathrm{A}}, \operatorname{IR} 1_{\mathrm{B}}\right)=(0,1)$.

In addition, $\beta_{\mathrm{i}}$ values (where $\mathrm{i}=0,1_{\mathrm{A}}, 1_{\mathrm{B}}, 2 \sim 5$ ) indicated the regression coefficients for each IR sign and age factor under this multivariate regression model. IR2 to IR5 in 
this model indicated the scales for IR2 to IR 5 signs of each lesion scored by the interpreting radiologists.

Further, we substituted the age and IR scales read by the radiologists into the ageadjusted multivariate regression model, thus obtaining the $\ln (\mathrm{OD})$ of each lesion. The cut-off values were derived and selected from the $\ln (\mathrm{OD})$ of this model. Under a given cut-off point or threshold, the $\ln (\mathrm{OD})$ of a lesion higher than or equal to the threshold implied a positive IR-test. For each given cut-off point, we also estimated the corresponding sensitivity, specificity, positive predictive value (PPV), negative predictive value (NPV), and Youden's Index (Index = sensitivity+specificity-1) $[17,18]$. The selection of cut-off points in our study was based on the criteria shown below:

For the cut-off values with sensitivity $>90 \%$, we selected one of them with highest corresponding specificity as the lowest cut-off value. Among those cut-off values with specificity $>90 \%$, we selected the one with highest corresponding sensitivity as the highest cut-off value. The cut-off value with the highest Youden's Index was also included in the analysis and was regarded as the most optimal cut-off point in our study. We also included other cut-off values between the highest and lowest cut-off values mentioned above, and a total of 5 cut-off values were taken for analysis.

We also evaluated the BI-RADS (Breast Imaging Reporting and Data System) categories [19] of lesions on mammography and ultrasound, and the corresponding true disease status (the histopathologic results). If the patient received only mammography or ultrasound, the BI-RADS ${ }^{\bullet}$ category of the lesion was determined by one of the imaging modalities; if the patient received both examinations, the BI-RADS ${ }^{\bullet}$ category was given based on higher concern between the two imaging modalities. Based on the most optimal cut-off point and the cut-off value with the highest sensitivity we selected, the corresponding sensitivity, specificity of IR imaging in each BI-RADS ${ }^{\bullet}$ category were also estimated. Since breast cancer may be inherently a life-threatening disease and the false-negativity (1-sensitivity) would cause severe consequences in clinical practice of breast imaging, therefore, we also included the selected cut-off value with highest sensitivity for detailed analysis.

Based on the age-adjusted multivariate model, the $\ln (\mathrm{OD})$ values of malignant and benign foci for all lesions were compared using Student's $t$-test. For the lesions with mammographic findings available for correlation, we categorized them into three major types of mammographic findings, that is, microcalcifications, microcalcifications associated with mass (or architectural distortion, focal asymmetry), or noncalcified lesions. We also investigated if there was any difference in $\ln (\mathrm{OD})$ values between malignant and benign lesions for different types of mammographic findings using Student's $t$-test or non-parametric Mann-Whitney U test.

We further stratified all lesions into three categories according to the pathologic size, that is, size larger than or equal to $2 \mathrm{~cm}$, size less than $2 \mathrm{~cm}$ but not less than $1 \mathrm{~cm}$, and size less than $1 \mathrm{~cm}$ across the largest diameter. We compared the $\ln (\mathrm{OD})$ values for lesions between benign and malignant lesions for these three categories by Student's $t$-test or Mann-Whitney $U$ test, in order to investigate the validity of the ageadjusted multivariate model in lesions with different size categories.

In our study, a $p$ value less than 0.05 was considered to show statistical significance. 


\section{Results}

In our study, a total of 298 lesions associated with 276 patients were excised with histopathological correlation. The clinical, conventional imaging, and pathological data are listed in Table 1. Of those, 174 lesions were malignant (DCIS, 22; invasive carcinoma, 152) and 124 lesions were benign (including $7 \mathrm{ADH}, 2 \mathrm{ALH}$ and 3 papillary lesions identified as high-risk lesions). The patients with malignant lesions tended to be older than those with benign lesions $(p<0.0001)$. The mean lesion size or extension of malignant lesions on preoperative conventional imaging studies and at surgical pathology was significantly larger than that of the benign lesions (Table 1). There were 215 lesions with available mammographic examinations for correlation. Of the lesions showing mammographic finding of microcalcifications, most of them were benign. Most of the lesions with the finding of microcalcifications with mass or noncalcified finding were malignant (Table 1).

Univariate logistic regression analysis of the diagnostic IR signs and the final results are shown in Table 2, and the malignant result was significantly associated with higher IR scores for all IR signs. However, for the age-adjusted multivariate regression analysis, only IR1, IR4 and IR5 remained statistically significant; and IR2 and IR3 turned out to be non-significant (Table 2). The Hosmer and Lemeshow test revealed a non-significant $p$ value ( $\mathrm{p}=0.496$ ), suggesting that the age-adjusted multivariate regression model was an acceptable, fitted model.

When investigating the diagnostic performance of each individual IR sign (Table 3), the IR5 revealed a highest AUC value among the five IR signs. However, the AUC of the age-adjusted multivariate regression model was 0.828 (Figure 5), which was higher than the AUC values of any of the individual IR signs; and its lower bound of 95\% CI was higher than the upper bound of the $95 \%$ CI of the AUC of all of the individual IR signs (Table 3). The intercept and regression coefficients of the age-adjusted multivariate regression model are shown in Table 3 . The selected cut-off values from the ageadjusted multivariate regression model with the derived sensitivities, specificities, PPV, NPV and Youden's Index are presented in Table 4. The highest Youden's Index was 0.49 (most optimal cut-off point), when the cut-off value was 0.3 with a sensitivity of $72.4 \%$, specificity of $76.6 \%$, PPV of $81.3 \%$ and NPV of $66.4 \%$ for the overall study population. According to the selection criteria we established, the lowest cut-off value of this model was -0.72 , with its corresponding sensitivity $92.0 \%$, specificity $44.3 \%$, PPV 69.9\% and NPV 79.7\%; the highest cut-off value was 1.27, and its corresponding specificity was $94.3 \%$ at a sensitivity of $50.0 \%$, PPV 92.6\%, NPV 57.4\%. The estimation of ln (OD) value for a malignant lesion and the corresponding IR, mammographic images are illustrated in Figures 3, 4.

When we used the $\ln (\mathrm{OD})$ value of 0.3 as the most optimal cut-off point, the corresponding sensitivity, specificity in each BI-RADS ${ }^{\circledR}$ category are shown in Table 5 . For the BI-RADS ${ }^{\oplus}$ category 3 lesions, IR imaging correctly identified the only 1 cancerous lesion (sensitivity 100\%) with a specificity of $75 \%(6 / 8)$. In BI-RADS ${ }^{\oplus}$ category $4 \mathrm{~B}$ findings, the IR imaging correctly identified highest proportion of true-negative lesions (specificity $84.6 \%, 33 / 39)$ compared with other categories but the sensitivity $(51.5 \%, 17 /$ 33) was somewhat lower than the other categories (Table 5).

On the other hand, when we used the -0.72 as the cut-off point, the sensitivity in each BI-RADS ${ }^{\oplus}$ category increased while the specificity decreased when compared with 
Table 1 Clinical and conventional imaging findings.

\begin{tabular}{llll}
\hline Basic findings & Malignant & Benign & $\boldsymbol{p}$ value \\
\hline Patient (lesion) number & $165(174)$ & $111(124)$ & \\
Age of patients (years) & $54.3($ SD 11.1) & $45.4(11.2)$ & $<0.0001^{\#}$ \\
Mammographic findings & $122^{\S}$ & 93 & $<0.0001^{\# \#}$ \\
Microcalcifications & 28 & 77 & \\
Calcifications+mass* & 40 & 3 & \\
Noncalcified lesion & 51 & 13 & $<0.0001^{\#}$ \\
Available ultrasound findings relevant & $125^{\S \S}$ & 64 & $0.001^{\#}$ \\
to the lesion sites & & $1.94(0.24-10)$ & \\
Imaging size**, cm (range) & $2.83(0.3-11)$ & $2.15(0.2-7.0)$ & \\
Pathologic size, cm (range) & $2.88(0.3-12)$ & &
\end{tabular}

*Lesions revealing calcifications associated with mass, focal asymmetry or architectural distortion. **Lesion size determined by the largest diameter between mammography and breast ultrasound.

s: 3 cancerous lesions were not shown on mammograms (false-negative).

\$\$: 2 cancerous lesions were negative on ultrasound.

Table 2 Analysis of IR signs and the final results by univariate and age-adjusted multivariate logistic regression.

\begin{tabular}{|c|c|c|c|c|c|}
\hline IR signs scores & $M$ & B & $p^{*}$ & $\begin{array}{l}\text { Univariate } \\
\text { \#OR(95\%Cl) } \\
\left(p^{* *}\right)\end{array}$ & $\begin{array}{l}{ }^{\# \#} \text { Multivariate } \\
{ }^{\#} \mathrm{OR}(95 \% \mathrm{Cl}) \\
\left(p^{* *}\right)\end{array}$ \\
\hline IR1 0 & 68 & 93 & $<0.0001$ & 1 & 1 \\
\hline 1 & 66 & 27 & & $\begin{array}{l}3.3(1.9-5.8) \\
(<0.0001)\end{array}$ & $\begin{array}{l}1.5(0.7-2.9) \\
(0.29)\end{array}$ \\
\hline 2 & 40 & 4 & & $\begin{array}{l}13.7(4.7-40.0) \\
(<0.0001)\end{array}$ & $\begin{array}{l}6.8(2.0-23.5) \\
(0.003)\end{array}$ \\
\hline IR2 0 & 70 & 78 & 0.0001 & 1 & 1 \\
\hline 1 & 104 & 46 & & $\begin{array}{l}2.5(1.6-4.0) \\
(0.0001)\end{array}$ & $\begin{array}{l}1.2(0.6-2.3) \\
(0.61)\end{array}$ \\
\hline $\mathrm{IR} 3^{\&}$ & 174 & 124 & - & $\begin{array}{l}1.4(1.2-1.7) \\
(<0.0001)\end{array}$ & $\begin{array}{l}1.2(0.95-1.4) \\
(0.13)\end{array}$ \\
\hline IR4 0 & 140 & 114 & $<0.01$ & 1 & 1 \\
\hline 1 & 34 & 10 & & $\begin{array}{l}2.8(1.3-5.8) \\
(<0.01)\end{array}$ & $\begin{array}{l}2.8(1.1-6.8) \\
(0.024)\end{array}$ \\
\hline IR5 0 & 38 & 77 & $<0.0001$ & 1 & 1 \\
\hline 1 & 136 & 47 & & $\begin{array}{l}5.9(3.5-9.8) \\
(<0.0001)\end{array}$ & $\begin{array}{l}2.8(1.4-5.8) \\
(0.005)\end{array}$ \\
\hline
\end{tabular}

M: malignant lesions; B: benign lesions; $\mathrm{Cl}$ : confidence interval.

\#OR: odds ratio; ${ }^{\#}$ multivariate: age-adjusted multivariate regression model.

\&: continuous variable.

$p: p$ value estimated by ${ }^{*}$ Chi-square test, ${ }^{*}$ logistic regression analysis.

Table 3 AUC values for each IR sign and for an age-adjusted multivariate logistic regression model.

\begin{tabular}{lll}
\hline model & AUC & $\mathbf{9 5 \% C l}$ \\
\hline Univariate & & \\
IR1 & 0.699 & $(0.639,0.758)$ \\
IR2 & 0.613 & $(0.549,0.678)$ \\
IR3 & 0.674 & $(0.611,0.736)$ \\
IR4 & 0.557 & $(0.492,0.623)$ \\
IR5 & 0.701 & $(0.640,0.763)$ \\
Multivariate & & \\
(age-adjusted) & 0.828 & $(0.783,0.873)$
\end{tabular}

Multivariate: age-adjusted multivariate regression model, which is shown as below: $\ln ($ odds $)=\ln (\mathrm{OD})=\alpha+\beta_{0}($ Age $)+\beta_{1 \mathrm{~A}}\left(\mathrm{IR} 1_{\mathrm{A}}\right)+\beta_{1 \mathrm{~B}}\left(\mathrm{IR} 1_{\mathrm{B}}\right)+\beta_{2}(\mathrm{IR2})+\beta_{3}(\mathrm{IR} 3)+\beta_{4}(\mathrm{IR} 4)+\beta_{5}(\mathrm{IR} 5)$

$=-5.463+0.0872($ Age $)+0.3783\left(\right.$ IR $\left.1_{\mathrm{A}}\right)+1.9157\left(\right.$ IR1 $\left.1_{\mathrm{B}}\right)+0.1728($ IR2 $)+0.1578($ IR3 $)+1.0278($ IR4 $)+1.0363($ IR5 $)$. 


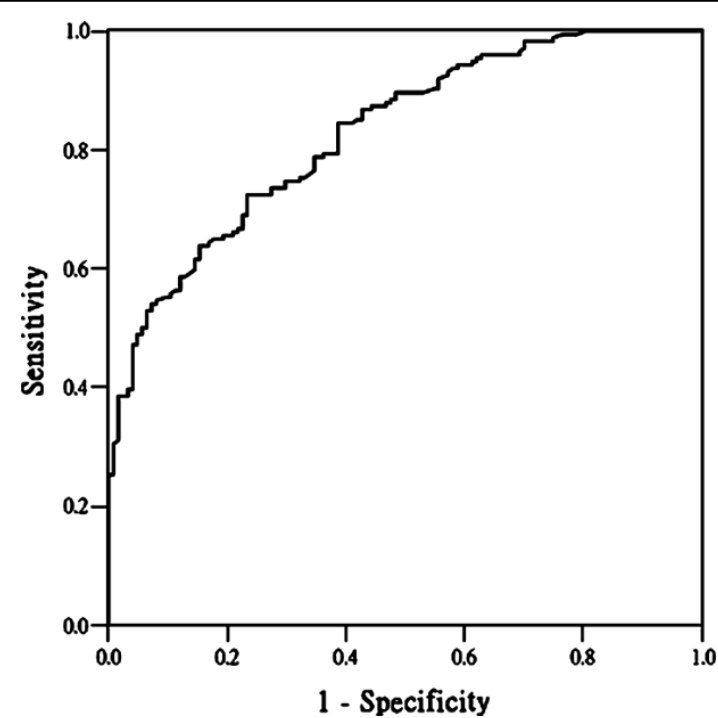

Figure $\mathbf{5}$ The ROC of an age-adjusted multivariate regression model. AUC $=0.828$.

the counterparts using 0.3 as the cut-off point (Table 5). Of them, in BI-RADS ${ }^{ø}$ category 3 findings, the IR imaging again can identify the only one cancerous lesion but the specificity dropped to $50 \%(4 / 8)$; the corresponding sensitivity of the BI-RADS category 4 A findings was lowest $(81.8 \%, 9 / 11)$ among all categories with a specificity of $36.7 \%(22 / 60)$. The specificity in BI-RADS ${ }^{\bullet}$ category $4 \mathrm{~B}(56.4 \%, 22 / 39)$ was higher than other categories with a sensitivity of $84.8 \%$ (28/33), and the sensitivities in BI-RADS ${ }^{\circ}$ categories $4 \mathrm{C}$ and 5 were higher than $90 \%$.

For all of the 298 lesions, the mean $\ln (\mathrm{OD})$ value of the malignant lesions (1.40) based on the age-adjusted multivariate model was higher than that of benign lesions (mean $\ln (\mathrm{OD})=-0.58)$ with statistically significant difference $(p<0.0001$, Student's $t$-test). We further estimated the $\ln (\mathrm{OD})$ values for lesions with mammographic findings available for correlation. For the lesions showing microcalcifications on mammograms, the mean $\ln (\mathrm{OD})$ value of malignant lesions (mean $=0.73$ ) was significantly higher than that of benign lesions (mean $=-0.4 ; p=0.001$, Student's $t$-test). For the lesions revealing calcifications with mass, the malignant lesions tended to have higher $\ln (\mathrm{OD})$ values

Table 4 The cut-off points, sensitivity, specificity and Youden's Index derived from an age-adjusted multivariate regression model.

\begin{tabular}{cccccc}
\hline Cut-off* & Sen (\%) & Spe (\%) & Youden & PPV (\%) & NPV (\%) \\
\hline 1.27 & 50.0 & 94.3 & 0.443 & 92.6 & 57.4 \\
0.82 & 62.6 & 84.7 & 0.473 & 85.2 & 61.8 \\
$0.30^{\#}$ & 72.4 & 76.6 & $* * 0.490$ & 81.3 & 66.4 \\
-0.30 & 85.6 & 57.3 & 0.429 & 73.8 & 74.0 \\
-0.72 & 92.0 & 44.3 & 0.363 & 69.9 & 79.7 \\
\hline
\end{tabular}

*Cut-off: cut-off values, derived from In(odds), which is the natural logarithm of the odds (OD) from the age-adjusted multivariate regression model. A lesion with a $\ln (\mathrm{OD})$ value higher than or equal to a given cut-off point is regarded as IR test-positive.

Sen: sensitivity; Spe: specificity; Youden: Youden's Index = sensitivity+specificity-1.

PPV: positive predictive value; NPV: negative predictive value.

** highest Youden's Index value ("the most optimal cut-off point we selected from the model).

The cut-off value was -3.51 when the sensitivity was $100 \%$ and the specificity $0 \%$; it was 5.47 when the sensitivity was $0.57 \%$ and specificity $100 \%$ (not shown in the table because they didn't meet the selection criteria of cut-off points in our study). 
Table 5 The correlation of BI-RADS $^{\oplus}$ categories on conventional imaging and the corresponding diagnostic performance of IR imaging based on the two cut-off points $(0.30$ and - 0.72)

\begin{tabular}{|c|c|c|c|c|c|c|c|c|c|}
\hline $\begin{array}{l}{ }^{\dagger} \mathrm{BI} \mathrm{RADS}^{\circledR} \\
\left({ }^{\mp} \mathrm{n}=281\right)\end{array}$ & $\begin{array}{l}\text { True } \\
\text { status (n) }\end{array}$ & $\begin{array}{l}\text { Cut-off (1) } \\
\ln (O D)\end{array}$ & & Sen(\%) & Spe(\%) & $\begin{array}{l}\text { Cut-off (2) } \\
\ln (O D)\end{array}$ & & Sen(\%) & Spe(\%) \\
\hline & & $>=0.30$ & $<0.30$ & & & $>=-0.72$ & $<-0.72$ & & \\
\hline \multirow[t]{2}{*}{$3(n=9)$} & $B(n=8)$ & 2 & 6 & 100 & 75.0 & 4 & 4 & 100 & 50.0 \\
\hline & $M(n=1)$ & 1 & 0 & & & 1 & 0 & & \\
\hline \multirow[t]{2}{*}{$4 \mathrm{~A}(\mathrm{n}=71)$} & $B(n=60)$ & 15 & 45 & 54.5 & 75.0 & 38 & 22 & 81.8 & 36.7 \\
\hline & $M(n=11)$ & 6 & 5 & & & 9 & 2 & & \\
\hline \multirow[t]{2}{*}{$4 B(n=72)$} & $B(n=39)$ & 6 & 33 & 51.5 & 84.6 & 17 & 22 & 84.8 & 56.4 \\
\hline & $M(n=33)$ & 17 & 16 & & & 28 & 5 & & \\
\hline \multirow[t]{2}{*}{$4 C(n=95)$} & $B(n=10)$ & 4 & 6 & 74.1 & 60.0 & 6 & 4 & 92.9 & 40.0 \\
\hline & $M(n=85)$ & 63 & 22 & & & 79 & 6 & & \\
\hline \multirow[t]{2}{*}{$5(n=34)$} & $B(n=2)$ & 2 & 0 & 81.3 & 0 & 2 & 0 & 100 & 0 \\
\hline & $M(n=32)$ & 26 & 6 & & & 32 & 0 & & \\
\hline
\end{tabular}

${ }^{\dagger} \mathrm{BI}^{-R_{A D S}}$ : BI-RADS $^{\oplus}$ category, an integrated BI-RADS category assessment based on conventional imaging (mammography and/or ultrasound), according to higher concern in any of or the two combined imaging modalities. ${ }^{\ddagger}$ There were 17 lesions from 17 patients having outside mammography and/or ultrasound and the detailed statements of $\mathrm{BI}-\mathrm{RADS}^{\oplus}$ categories were not obtained, and thus leaving 281 lesions for evaluation. $\mathrm{BI}^{-\mathrm{RADS}^{\circledR}}{ }^{\circ}$ Category 3: probably benign finding.

$\mathrm{BI}^{-R_{A D S}}{ }^{\oplus}$ Category $4 \mathrm{~A}$ : a lesion with low suspicion level for malignancy.

BI-RADS $^{\circledast}$ Category 4B: a lesion with intermediate concern for malignancy.

BI-RADS $^{\circledast}$ Category 4C: a lesion with moderate concern for malignancy.

BI-RADS $^{\oplus}$ Category 5: a lesion which is highly suggestive of malignancy.

$\mathrm{B}$ : benign (including high-risk lesions); $\mathrm{M}$ : malignant.

Cut-off (1): the cut-off value of 0.30; cut-off (2): the cut-off value of -0.72 .

$\ln (O D)>=$ the selected cut-off value indicated IR-test positive, and < the cut-off value indicated IR-test negative; In(OD): In(odds).

Sen: sensitivity = (true-positive number)/(the number of malignant disease status); while true- positive indicated IR-test positive as well as disease positive (malignant).

Spe: specificity = (true-negative number)/(the number of benign disease status); while true-negative indicated IR-test negative as well as disease negative (benign or high risk lesions).

$($ median $=1.01)$ than benign lesions (median $=-0.63)$ with statistically significant difference ( $p<0.01$, Mann-Whitney $\mathrm{U}$ test). For lesions with noncalcified findings, the malignant lesions again tended to have higher $\ln (\mathrm{OD})$ values (median $=1.35)$ than benign lesions (median $=0.23 ; p=0.002$, Mann-Whitney $\mathrm{U}$ test).

For the lesions stratified by different pathologic size categories, the $\ln (\mathrm{OD})$ values of the malignant lesions tended to be higher than those of benign lesions with statistically significant difference in all three categories (Table 6).

\section{Discussion}

Though infrared imaging of the breast is not widely used for various reasons, it has been reported to show promising results in some series [6,7,9-12,15,20]. Parisky, et al [6] used computerized IR imaging for suspicious findings on mammograms, and reported that IR imaging featured a relatively high sensitivity (97-99\%) and negative predictive value (95-99\%) and thus can differentiate benign from malignant lesions reliably. However, the reported specificity in this series ranged from 14 to $18 \%$, which was relatively low. Keyserlingk, et al [7] reported that the sensitivity of mammography alone was $85 \%$ and that of combined modalities of digital IR, mammography was $95 \%$. Thus, digital IR imaging can provide additional information for breast lesion diagnosis. There were some authors from different series who used an artificial neural network, computer software, or segmentation technique for breast cancer detection, monitoring of treatment response, and for establishing an interpretive model $[3,5,11,12,15,20]$. 
Table 6 The $\ln ($ odds) values $(\ln (O D))$ between benign and malignant lesions stratified by size

\begin{tabular}{llll}
\hline & $\mathbf{M}^{\dagger}$ & $\mathbf{B}^{\mathbf{*}}$ & $\boldsymbol{p}$ \\
\hline $\operatorname{size}^{*}(\mathrm{~cm})>=2$ & 111 & 51 & $<0.001^{\#}$ \\
$\mathrm{n}$ & $1.65^{\mathrm{a}}$ & $-0.61^{\mathrm{a}}$ & $<0.001^{\#}$ \\
$\ln (\mathrm{OD})$ & & 27 & \\
$1<=\operatorname{siz}{ }^{*}(\mathrm{~cm})<2$ & 46 & $-0.74^{\mathrm{a}}$ & \\
$\mathrm{n}$ & $1.20^{\mathrm{a}}$ & & $<0.028^{\S}$ \\
$\ln (\mathrm{OD})$ & 17 & 46 & $-0.62^{\mathrm{m}}$ \\
$\operatorname{size}(\mathrm{cm})<1$ & $0.36^{\mathrm{a}}$ & & \\
$\mathrm{n}$ & & & \\
$\ln (\mathrm{OD})$ & & & \\
\hline
\end{tabular}

${ }^{\dagger} \mathrm{M}$ : malignant; ${ }^{\ddagger} \mathrm{B}$ : benign (including high-risk lesions); $\ln (\mathrm{OD})$ : $\ln (\mathrm{odds})$.

*size: determined by the pathologic size (across the largest diameter).

a: Average, mean $\ln (O D)$.

$\mathrm{m}$ : Median $\ln (\mathrm{OD})$.

\#: Student's $t$-test; ${ }^{\S}$ : Mann-Whitney $\mathrm{U}$ test.

However, though digital IR imaging uses the combined criteria of temperature and vascular pattern for diagnosis, the diagnostic criteria are still somewhat variable according to the aforementioned series $[3,5,7,11,12,15,20]$. In our study, for a subpopulation with diagnostic purpose, we tried to establish an age-adjusted multivariate regression model to predict breast cancer disease status based on IR findings, and to investigate the diagnostic performance of IR imaging based on this model.

For univariate analysis of the different diagnostic IR signs in our study, we found that there was a significant association between malignancy and higher IR scores for each IR sign, and the IR5 (asymmetric vascular pattern at lesion site compared to the contralateral side) showed a higher AUC value than other signs. This was not surprising, since IR imaging of the breast was designed to detect temperature elevations of the tumor, and the site with elevated surface temperature will indeed cause an asymmetric thermographic pattern. Therefore, though the IR1 sign has a somewhat similar implication as IR5, it is a quantitative measure, while IR5 is a morphologically descriptive sign. In addition, for a better comparison of the lesion site with the contralateral breast, we excluded women who had breast surgery previously, to eliminate the inherent asymmetric thermographic pattern due to parenchymal loss after partial or total mastectomy.

In age-adjusted multivariate regression analysis, the malignant result was associated with higher IR scores, especially for IR1, IR4 and IR5 when adjusted for other factors. Indeed, this model revealed a better diagnostic performance when compared with each IR sign from univariate analysis, based on a diagnostic population in our study. Though there were some series reporting the diagnostic value of IR imaging for breast cancer screening $[1,4,9,21,22]$, and abnormal IR imaging of the breast was reported to be a cancer risk predictor [9], IR imaging has not been routinely used for screening purpose. Our study, like some of other series $[6,7,11]$, investigated the diagnostic efficacy of IR imaging for specific groups with inconclusive or suspicious findings on conventional imaging modalities (mammography or ultrasound). In addition, most of these reported series combined the mammographic, clinical breast examination and IR together to discuss the diagnostic values of IR as an adjunct tool. But these series seldom included ultrasonographic correlation [6-8,11]. However, in our study, there was a considerable proportion of participants that had mammographic and ultrasonographic 
correlations, and a combined analysis of both imaging modalities would provide a more convincing diagnostic result than if only one of the modalities was available. Therefore, we designed our study so that the interpreting radiologists were informed of only the lesion site and size on conventional imaging modalities when reading the IR images, and were blinded to the clinical history and detailed mammographic and ultrasonographic findings, and the pathological results. We established this protocol to ensure a more objective reading of IR images. This allowed us to establish an interpretive model for IR imaging of the breast with less bias. After establishing an appropriate interpretive model, it would be possible to apply this model or similar methodology to other groups, such as a screening population.

In our study, the cut-off values of the IR imaging were estimated from the ageadjusted multivariate regression model, which also derived the AUC of the ROC curve for diagnostic performance evaluation, and the various sets of sensitivity and specificity values can be inferred from different cut-off values. There is always a trade-off between sensitivity and specificity, since a higher sensitivity will be accompanied with a lower specificity and vice versa. The above observations also apply to the analysis of PPV and NPV. As can be seen in Table 4, cut-off values with higher PPV and specificity correspond to a lower NPV and sensitivity, and vice versa. The strategy of which arm should be stressed more depends on the purpose of the test and the target population enrolled $[17,18,23]$. Under this consideration, the cut-off value with the highest Youden's Index might not be the most optimal threshold value $[17,18,23]$. For the lowest selected cut-off point (sensitivity $92.0 \%$ and specificity $44.3 \%$ ) in our study, it showed a relatively high sensitivity and an acceptable specificity when compared with other reported series $[6,11]$. However, in BI-RADS ${ }^{\bullet}$ category 3 findings, the cut-off point with highest Youden's Index (cut-off value 0.3) yielded a high sensitivity and moderate specificity (75\%), but the specificity dropped to $50 \%$ when using the -0.72 as the cutoff point. Therefore, for the IR imaging, the cut-off value of 0.3 was a more optimal cut-off point for probably benign findings as an adjunct role in diagnostic breast imaging. And for the category 4A and 4B findings, though the sensitivities using the -0.72 as the cut-off point increased as compared with the cut-off point of 0.3, the values $(81.8 \%$ in $4 \mathrm{~A}$ and $84.8 \%$ in $4 \mathrm{~B}$; Table 5$)$ were still not satisfactory in a diagnostic population. However, if we read the IR imaging after viewing the detailed mammographic and ultrasound images, the diagnostic performance may be different and should be likely elevated somewhat compared with our current study design. In that situation, the clinical role of IR imaging may be more enhanced at the help with decision making when the mammography or ultrasound shows ambiguous findings.

In our study, for the lesions with mammographic findings available for correlation, we found that the malignant lesions tended to have higher $\ln (\mathrm{OD})$ values ( $\ln$ (odds) values) than benign lesions in all three types of mammographic findings.

When we compared the $\ln (\mathrm{OD})$ values between the benign and malignant lesions stratified by different pathologic size categories, we found that the malignant lesions tended to have higher $\ln (\mathrm{OD})$ values than benign lesions in all three categories, even for lesions less than $1 \mathrm{~cm}$ and $2 \mathrm{~cm}$ in size. The findings further ensure the validity of application of the age-adjusted multivariate model to lesions with smaller tumor size.

There are some limitations to our study. First, as we stated previously, our study participants were for diagnostic purpose, and we interpreted the IR images referring to the 
lesion site and size, that is, a targeted interpretation. Therefore, currently we have not yet documented the diagnostic value of our model for screening purposes. Second, for the purpose of keeping the statistical efficiency of the model analysis, we categorized 12 high-risk lesions into the benign group due to the limited sampling size, and we didn't further categorize the malignant lesions into non-invasive and invasive, or different grades of carcinomas. Therefore, the diagnostic performance of IR imaging in high-risk lesions or different pathologic grades of carcinomas may not be known. Finally, we excluded patients having a past history of breast surgery to reduce the interpretation bias caused by asymmetric thermographic pattern due to parenchymal loss, as we mentioned above. Thus, we haven't applied our interpretative model to post-operative breasts.

\section{Conclusions}

In conclusion, IR imaging of the breast is a noninvasive diagnostic examination. We established an age-adjusted multivariate logistic regression model under a specific clinical setting for diagnostic purpose. However, its values for post-operative breasts, for screening populations, and for high-risk lesions or different grades of breast carcinomas have not been verified in our study and should be further investigated in the future. Further, from our study, it has not yet been proven whether the IR imaging would reliably avoid unnecessary biopsy for suspicious findings on mammograms and breast ultrasound.

\section{Acknowledgements}

The authors thank Dr. Shin-Liang Pan for help with statistical analysis.

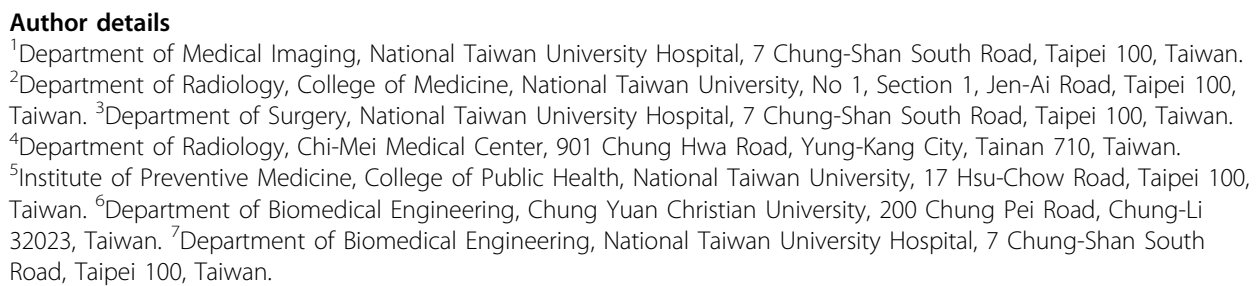

\section{Authors' contributions}

JW participated in the design of the study, imaging interpretation and carried out the statistical analysis, drafted and revised the manuscript. KJC participated in the design of the study, the acquisition of the data, and helped to revise the manuscript. CYC participated in the design of the study, imaging interpretation and carried out the statistical analysis, and revised the manuscript. KLC participated in the statistical analysis and helped to revise the manuscript. YST participated in the design of the study, the acquisition of the data, and helped to revise the manuscript. YMW participated in the design of the study, the acquisition of the data, imaging interpretation, and helped to revise the manuscript. YCT participated in the acquisition of the data and helped to revise the manuscript. TTFS participated in the design of the study, carried out the statistical analysis, drafted and revised the manuscript. All authors read and approved the final manuscript.

\section{Competing interests}

The study was supported by AG Digital Technology Corporation, Taipei, Taiwan.

The organization supplied the thermographic machine and funding of the study. However, the article-processing charge is not supported by the organization.

Received: 26 August 2009

Accepted: 7 January 2010 Published: 7 January 2010

\section{References}

1. Head FJ, Wang F, Lipari CA, Elliott RL: The important role of infrared imaging in breast cancer. IEEE Engineering In Medicine And Biology 2000, 19:52-57.

2. Foster KR: Thermographic detection of breast cancer. IEEE Engineering In Medicine And Biology 1998, 17:10-14. 
3. Arena F, Barone C, Dicicco T: Use of infrared imaging in enhanced breast cancer detection and monitoring of the clinical response to treatment. Proceedings of the 25th Annual International Conference of the IEEE EMBS: 17-21 September 2003; Cancun, Mexico Edited by Engineering in Medicine and Biology Society 2003, 1129-1132.

4. Threatt B, Norbeck JM, Ullman NS, Kummer R, Roselle PF: Thermography and breast cancer: an analysis of a blind reading. Annals N Y Acad Sci 1980, 335:501-519.

5. Ng EYK, Sudharsan NM: Computer simulation in conjunction with medial thermography as an adjunct tool for early detection of breast cancer. BMC Cancer 2004, 4:17, Published online 2004 April 28.

6. Parisky YR, Sardi A, Hamm R, Hughes K, Esserman L, Rust S, Callahan K: Efficacy of computerized infrared imaging analysis to evaluate mammographically suspicious lesions. AJR 2003, 180:263-269.

7. Keyserlingk JR, Ahlgren PD, Yu E, Belliveau N: Infrared imaging of the breast: initial reappraisal using high-resolution digital technology in 100 successive cases of stage I and II breast cancer. The Breast J 1998, 4:245-251.

8. Lapayowker MS, Revesz G: Thermography and ultrasound in detection and diagnosis of breast cancer. Cancer 1980, 46:933-938.

9. Gautherie M, Gros CM: Breast thermography and cancer risk prediction. Cancer 1980, 45:51-56.

10. Ohsumi S, Takashima S, Aogi K, Usuki H: Prognostic value of thermographical findings in patients with primary breast cancer. Breast Cancer Res and Treat 2002, 74:213-220.

11. Arora N, Martins D, Ruggerio D, Tousimis E, Swistel AJ, Osborne MP, Simmons RM: Effectiveness of a noninvasive digital infrared thermal imaging system in the detection of breast cancer. Am J Surg 2008, 196:523-526.

12. Ng EYK, Kee EC: Advanced integrated technique in breast cancer thermography. J Med Eng Technol 2008, 32:103114.

13. Gautherie M: Improved system for the objective evaluation of breast thermograms. Prog Clin Biol Res 1982, 107:897905.

14. Hobbins WB: Thermography of the breast- a skin organ. Thermal assessment of breast health Lancaster, UK: MTP Press LtdGautherie M, Albert E, Keith L 1983, 40-48.

15. Ng EYK, Ung LN, Ng FC, Sim LSJ: Statistical analysis of healthy and malignant breast thermography. J Med Eng Technol 2001, 25:253-263.

16. Isard HJ: Thermographic "edge sign" in breast carcinoma. Cancer 1972, 30:957-963.

17. Hilden J, Glasziou P: Regret graphs, diagnostic uncertainty and Youden's Index. Statistics in Medicine 1996, 15:969986.

18. Youden WJ: Index for rating diagnostic tests. Cancer 1950, 3:32-35.

19. American College of Radiology: Breast imaging reporting and data system (BI-RADS ${ }^{\odot}$ ). Reston, VA , 42003.

20. Ng EYK, Chen Y, Ung LN: Computerized breast thermography: study of image segmentation and temperature cyclic variations. J Med Eng Technol 2001, 25:12-16.

21. Haberman JD, Love TJ, Francis JE: Screening a rural population for breast cancer using thermography and physical examination techniques: methods and results-a preliminary report. Ann NY Acad Sci 1980, 335:492-500.

22. Isard HJ: Thermograhy in mass screening of cancer: success and failures. Ann NY Acad Sci 1980, 335:489-491

23. Hausen H: Caries prediction- state of the art. Community Dent Oral Epidemiol 1997, 25:87-96.

doi:10.1186/1475-925X-9-3

Cite this article as: Wang et al: Evaluation of the diagnostic performance of infrared imaging of the breast: a preliminary study. BioMedical Engineering OnLine 2010 9:3.

\section{Submit your next manuscript to BioMed Central and take full advantage of:}

- Convenient online submission

- Thorough peer review

- No space constraints or color figure charges

- Immediate publication on acceptance

- Inclusion in PubMed, CAS, Scopus and Google Scholar

- Research which is freely available for redistribution 